\title{
TNF Antagonists Opened the Way to Personalized Medicine in Rheumatoid Arthritis
}

\author{
Ferry Breedveld \\ Leiden University Medical Center, Leiden, The Netherlands
}

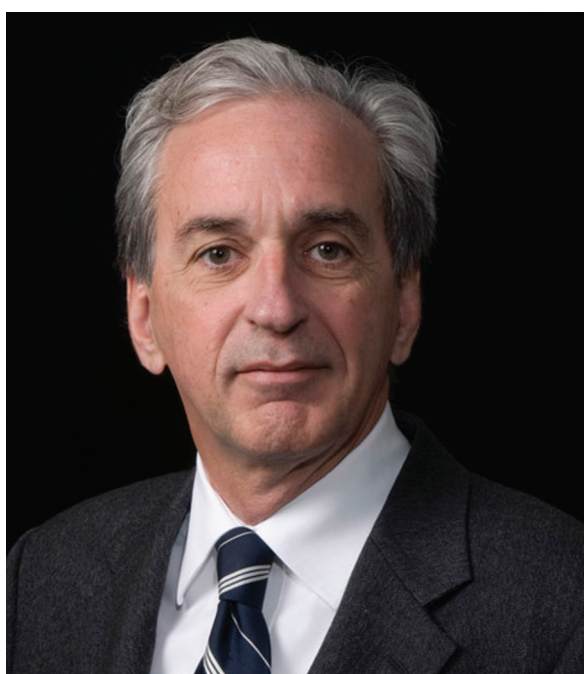

\begin{abstract}
Rheumatoid arthritis (RA) is an autoimmune disease resulting from a largely unknown interaction between genetically determined and environmental factors. Progress in the understanding of this chronic inflammation in the synovial lining of joints has led to the insight that one cytokine, tumor necrosis factor (TNF), has an important role. This insight started the development of a series of targeted and highly effective therapeutics for RA and a range of other autoinflammatory diseases. RA has changed from a severely debilitating disease into a disease where progression can be stopped in most of the patients.
\end{abstract}

Online address: http://www.molmed.org

doi: $10.2119 / \mathrm{molmed} .2014 .00168$

\section{INTRODUCTION}

Rheumatoid arthritis (RA) is a severe disease frequently resulting in disability. This is caused in the short term by joint pain and swelling. Over the long term, disability is caused by deformation resulting from joint destruction. Approximately $0.5 \%$ of the population develops this chronic destructive inflammation in the synovial membrane of joints. When I started rheumatology training in 1980, wheelchairs dominated the view of the rheumatology clinic and patients were admitted with gross skeletal destruction and signs of severe systemic inflammation. This picture has improved dramatically. RA patients now are seldom admitted, maintain relatively good mobility and enjoy a much better quality of life.

The largest contribution to this great success in medicine is the introduction of TNF antagonists in the 1990s. Rational treatment of RA started in 1897 when the German chemist Hoffman synthesized acetylsalicylic acid. Aspirin and its modern derivatives are helpful agents for symptomatic relief. In 1949, Philip Hench reported rapid improvement in RA patients after administration of cortisone. As a result, he received the Nobel Prize in 1950. However, the use of these agents declined in the 1980s due to the recognition of side effects. In the second half of the last century, several therapeutic agents were registered for RA treatment

Address correspondence to Ferry Breedveld, Leiden University Medical Center, Leiden, The Netherlands. Phone: +31-(0)651836838; Fax: +31-(0)715248126; E-mail: f.c.breedveld@lumc.nl. Submitted August 26, 2014; Accepted for publication September; Published Online (www.molmed.org) December 16, 2014.

\section{The Feinstein Institute for Medical Research Empowering Imagination. Pioneering Discovery.

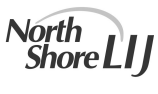

based on their potential to suppress symptoms and inhibit structural damage. They include antimalarial drugs, sulphasalazine and leflunomide. Of these, low dose methotrexate became the most widely used due to its favorable efficacy and toxicity profile.

\section{HISTORY OF ANTI-TNF TREATMENT IN RA}

Many rheumatologists are aware of the fact that triumphs in basic science create the basis for TNF blockade in RA. Tony Cerami studied cachexia in cattle infected with parasites. He found that macrophages in vitro produced a cytokine in response to infectious agents. That cytokine could elicit in noninfected animals most of the sequelae observed in infected animals (1). Subsequently he wrote a United States patent describing the use of antibodies to this protein, then named cachectin, in diseases where excessive cachectin production could be responsible for the damage, including rheumatoid arthritis. Others were able to show that this protein in experimental animals was almost identical to that of a human cytokine named TNF. The utility 
of neutralizing the bioactivity of TNF with antibodies was subsequently studied extensively in many animal models of disease. The role of TNF in RA was then expanded by Feldman and Maini (2). In March of 1992 they showed that infliximab, a chimeric anti-TNF monoclonal antibody, was highly effective in suppressing signs and symptoms of inflammation in RA patients (3).

For me, it was a great pleasure to participate in the subsequent clinical studies that showed the nearly complete suppression of joint damage progression and the relative favorable safety of TNF blockade (4). The use of monoclonal antibodies and TNF receptor-Fc fusion proteins long term in a disease with a relatively high prevalence has had considerable impact on the pharmaceutical industry as judged by many new products that have now entered clinical trials or already received access to the market.

\section{OPTIMAL USAGE OF ANTI-TNF IN RA}

The availability of an effective intervention in RA included the development of a completely different diagnostic therapeutic algorithm. First, more objective tools were developed to monitor disease activity and progression of destruction; then criteria were refined to diagnose early RA. Subsequent clinical studies in the last decade provided solid clinical evidence for the following changes in the treatment paradigm.

1. Very early recognition and treatment after diagnosis suppresses the progression of joint destruction more rigorously than delayed introduction (5).

2. Combination therapy, particularly anti-TNF and methotrexate, is more effective than monotherapy (6).

3. Frequent evaluation of disease activity with frequent therapy adjustments striving for low disease activity results in better disease outcomes than "routine" care (7).

4. Patients with active RA who do not respond to methotrexate treatment in 3 months should be treated with a TNF blocker (6).
5. Patients who fail to respond to TNF antagonists can be treated with another anti-TNF or another biologic agent (8).

\section{THE BeST STUDY}

In 1998, the rheumatology group in Leiden designed a study that would become one of the most referred-to papers of this clinical field: The BeSt study (acronym for Behandel Strategieën, translated as "treatment strategies") (6). This observational study provided many insights that became the scientific bases of the treatment guidelines of today.

The BeSt study introduced a new study design by comparing four different treatment strategies in patients with recent onset RA. Treatment adjustments within these strategies were determined by regular assessments of disease activity at 3-month intervals. In case of an insufficient response, patients proceeded to the next step. The strategies were: 1) sequential monotherapy; 2) step-up combination therapy; 3) initial combination therapy; and 4) initial therapy with methotrexate and infliximab (a TNFblocking monoclonal antibody).

The clinical observation led to the following conclusions: (a) relief of pain and clinical symptoms as well as functional improvement started more quickly and was larger in patients who started with infliximab and methotrexate (6); (b) therapy had to be adjusted less frequently in patients starting with combination therapy: after one year, $72 \%$ of the patients starting with infliximab were still on the first treatment step, significantly more frequent than in the other arms (6); (c) progression of radiologic damage was significantly less frequent in patients treated with early combination therapy (6); (d) tight disease control and immediate adjustment of medication has a better clinical outcome than "routine" practice (9); (e) patients who received remission drugs could be tapered successfully in $\pm 50 \%$ of the patients treated with infliximab which was significantly more frequent than in other therapies (6); (f) all treatment arms have the same safety profile (6); (g) the majority of all patients improved; however, patients who had received initial combination therapy with prednisone were less satisfied than patients who had received initial combination therapy with infliximab (10); (h) savings on productivity could compensate for the extra costs of infliximab therapy (11); and (i) patients treated early with infliximab + methotrexate responded better than patients who received that combination in a later stage of the disease (12).

This observation about better reponse from patients treated early with infliximab + methotrexate suggests a window of opportunity where early powerful therapies result in long-lasting remission.

The BeSt study established that RA patients benefit from an early diagnosis and a dynamic treatment strategy in which treatment strategies are continuously being adjusted. Furthermore the clinical state of remission, hardly discussed prior to 1990, now seems to have become a realistic goal for most of RA patients.

\section{RHEUMATOLOGY IN 2014}

The introduction of TNF antagonists heralded a new era in the treatment of RA. In most countries, this treatment is reimbursed, as anti-TNF ranks in the top of the list of expensive treatments. This is justified given the positive results of cost utility analyses (11). On average, $25 \%$ of all RA patients are being treated with a biologic agent and the success is astonishing with early and intensive treatment. The disease can be brought into remission or very low disease activity in $\pm 70 \%$ of the patients. Around $20 \%$ of the patients maintain this therapeutic success even following discontinuation of maintenance therapy. The success of TNF blockade in RA led to trials in other diseases in which TNF was known to be expressed. There have been successful trials and subsequent approval in juvenile rheumatoid arthritis, ankylosing spondylitis and psoriasis. With all these new treatment possibilities, rheumatology has become a prestigious and attractive specialty for young doctors. They 
have the option to train in clinical science and contribute to the field of translational medicine. Yet, despite all these improvements, there still is an unmet need for patients and room for further therapeutic improvement. An important goal for the future is increasing the worldwide availability of this costly treatment.

\section{CONCLUSION}

The fact that work from Tony Cerami on parasites in cattle led to the discovery of a molecule responsible for several destructive aspects of chronic inflammation; the surprising observation of the inhibition of one molecule in a complex heterogeneous and multigenic disease such as RA is so therapeutic; and the solid clinical research that brought all health care systems to accept this expensive therapy and improve the quality of life of a large number of patients can only reaffirm the conclusion that science can change the world. The history of TNF blockade is the most impressive experience in my professional life. I hope that rheumatology can create another historical event of similar importance.

\section{DISCLOSURE}

The authors declare that they have no competing interests as defined by Molecular Medicine, or other interests that might be perceived to influence the results and discussion reported in this paper.

\section{REFERENCES}

1. Tracey KJ, Vlassara H, Cerami A. (1989) Cachectin/ tumor necrosis factor. Lancet. 333:1122-6.

2. Brennan FM, Jackson A, Chantry D, Maini R, Feldmann M. (1989) Inhibitory effect of TNF $\alpha$ antibodies on synovial cell interleukin-1 production in rheumatoid arthritis. Lancet. 334:244-7.

3. Elliott MH, et al. (1993) Treatment of rheumatoid arthritis with a chimeric monoclonal antibody to tumor necrosis factor $\alpha$. Arthritis Rheum. 36:1681-90.

4. Lipsky PE, et al. (2000) Infliximab and methotrexate in the treatment of rheumatoid arthritis. N. Engl. J. Med. 343:1594-602.

5. Van Dongen H, et al. (2007) Efficacy of methotrexate treatment in patients with probable rheumatoid arthritis: a double blind, randomized, placebo-controlled trial. Arthritis Rheum. 56:1424-32.

6. Goekoop-Ruiterman YP, et al. (2007) Comparison of treatment strategies in early rheumatoid arthritis: a randomized trial. Ann. Intern. Med. 146:406-15.

7. Verstappen SM, et al. (2007) Intensive treatment with methotrexate in early rheumatoid arthritis; aiming for remission. Ann. Rheum. Dis. 66:1443-9.

8. Smolen JS, Aletaha D, Koeller M, Weisman MH, Emery P. (2007) New therapies for treatment of rheumatoid arthritis. Lancet. 370:1861-74.

9. Goekoop-Ruiterman YPM, et al. (2010) DAS driven therapy versus routine care in patients with recent onset active RA. Ann. Rheum. Dis. 69:65-69.

10. Goekoop-Ruiterman YPM, et al. (2007) Patient preferences for treatment: report from a randomized comparison of treatment strategies Ann. Rheum. Dis. 66:1227-32

11. Van den Hout WB, et al. (2009) Cost utility analysis of treatment strategies in patients with early rheumatoid arthritis. Arthritis Rheum. 61:291-9.

12. Van der Kooy SM, et al. (2009) Clinical and radiological efficacy of initial versus deluged treatment with infliximab plus methotrexate in patients with early rheumatoid arthritis Ann. Rheum. Dis. 68:1153-8. 